\section{Ultraviolet or blue- filtering intraocular lenses: what is the evidence?}

SM Downes

\begin{abstract}
Cataract surgery was revolutionised by the introduction of modern intraocular lenses in the late 1940's. By the late 1960's to 1970's evidence had emerged that short-wavelength light caused phototoxicity at the retina and retinal pigment epithelium. By the early 1980's ultraviolet filters had been incorporated into intraocular lenses. This caused intense controversy, as there was concern that the UV-filtering chromophore might leach out into the eye causing toxicity. With the arrival of blue-filtering intraocular lenses (BFIOLs) in 1990's, a further debate was ignited as to their safety and potential

publications have reported on the potential benefits and pitfalls of implanting a bluefiltering lens. The potential disadvantages raised in the literature over the last 25 years since their introduction, regarding compromise of visual function and disruption of the circadian system, have been largely dispelled. The clear benefits of protecting the retina from short-wavelength light make a BFIOLs a sensible choice. The purpose of this article presented at the Cambridge symposium 2015 is to review the literature on this subject.

Eye (2016) 30, 215-221; doi:10.1038/eye.2015.267; published online 8 January 2016
\end{abstract} disadvantages. Selecting the optimal performing intraocular lens to obtain the best visual performance with the fewest potential drawbacks has become complex and challenging for cataract surgeons and their patients with the wide choice of lenses available. Choosing a personalised lens to address astigmatism, presbyopia, spherical aberration, chromatic aberration, and potentially to shield the retina from shortwavelength light is now possible. The potential benefits and possible side effects of these different innovations emphasise the importance of assessing the evidence for their clinical utility, allowing the surgeon and the patient to weigh-up the risk benefit ratio and make an informed decision. The BFIOLs were developed to reduce cyanopsia, address chromatic aberration, and improve contrast sensitivity in different lighting conditions, as well as to prevent short-wavelength light reaching the retina thus potentially reducing the risk of developing age-related macular degeneration. Further design development of the BFIOLs was to mimic the natural crystalline lens absorption and transmittance properties in adulthood. Multiple

\section{Introduction}

The introduction of blue-filtering intraocular lenses (BFIOLs) for implantation after cataract surgery is still the subject of active debate, with the publication of $\sim 100$ articles focusing on the potential advantages and disadvantages of BFIOLs compared with ultraviolet filtering lenses (UVFIOLs). ${ }^{1-5}$ BFIOLs are also referred to as yellow- or orange-tinted intraocular lenses (IOLs). Historically, the impetus to develop BFIOLs was to reduce chromatic aberration, and cyanopsia, where the recipient of the IOL notices a blue tinge to their vision post surgery, ${ }^{3}$ and to provide protection against short-wavelength light because of the evidence that shortwavelength light is toxic to the retina and retinal pigment epithelium. (Van Norren-Cambridge Symposium Paper $)^{6,7}$ It was postulated that reduction or elimination of short-wavelength light to the retina by implanting a BFIOL could prevent the development of age-related macular degeneration. ${ }^{8}$ Other suggested benefits included improvement in contrast sensitivity ${ }^{9}$ and reduction in glare in certain lighting
Oxford Eye Hospital, Oxford University Hospitals Foundation Trust, and Nuffield Laboratory of Ophthalmology, University of Oxford, Oxford, UK

Correspondence:

SM Downes, Ophthalmology, University of Oxford, Oxford Eye Hospital, Oxford University Hospitals Foundation Trust, John Radcliffe, West Wing, Headley Way, Oxford OX3 9DU, UK

Tel: +44 1865 234567;

Fax: +44 1865234515 .

E-mail: susan.downes@ eye.ox.ac.uk

Received: 25 November 2015 Accepted: 25 November 2015 Published online:

8 January 2016 
conditions. ${ }^{10,11}$ Another benefit claimed for the BFIOL includes a reduction in blood retinal barrier disruption. ${ }^{12}$ It is interesting to note that several fish including the masked greenling show changeable colouration of their corneas from deep yellow to red when exposed to bright light and become colourless in the dark; this property is conferred by reversible pigment in the corneas. ${ }^{13}$ This is thought to reduce light dispersion in the short-wave region of the spectrum, enhance the visual contrast, and protect from bright illumination. Skiers and sharp shooters often wear yellow lenses to cut down glare, and reduce chromatic aberration. ${ }^{9}$ More recently, other innovative IOL lens designs have included the development of an IOL called a phototropic IOL that is clear in the absence of UV radiation and turns yellow in the presence of UV light. This system would be expected to provide optimal protection from short-wavelength blue light during the day and turns clear in low lighting conditions. ${ }^{14}$ Theoretical disadvantages of implanting a BFIOL compared with a UVFIOL have been raised over the last 25 years. The main concerns are that implantation of a BFIOL will lead to altered colour perception, reduced colour vision, a reduction in scotopic visual function, reduced contrast sensitivity in mesopic and scotopic conditions, and disruption of circadian photoentrainment. ${ }^{15,16}$

There have been excellent comprehensive articles reviewing this subject in detail and a more recent metaanalysis by Zhu in 2012. ${ }^{1-5}$ Since then more information has become available regarding the impact of the BFIOL on the sleep-wake cycle, ${ }^{17,18}$ blood pressure, ${ }^{19}$ mood, ${ }^{20}$ and cognitive function ${ }^{18}$ as well as early small studies looking at age-related macular degeneration (AMD) development. ${ }^{21,22}$

\section{Photoprotection and photoreception}

Transmittance and absorption properties of BFIOLs and UVFIOLs have been compared with each other, as well as to the natural crystalline lens. Artigas ${ }^{23}$ and co-authors show that not all UV filters incorporated into UVFIOLs offer equal protection from UV light. They go on to state that the filters that provide greater photoprotection against UV radiation, and blue light, are yellow and orange, and that yellow and orange IOL filters may be best suited for cases requiring special retinal protection. Van Norren and Kraats reported on spectral transmission of IOLs expressed as virtual age, which provides a useful method of comparison of different IOLs regarding their different short-wavelength transmission and absorption characteristics. ${ }^{24}$ They demonstrated that a UVFIOL mimics the lens transmission and absorption of a newborn ranging up to a teenager; whereas a BFIOL, depending on the lens type, is more typical of the transmission seen in early-to-late middle age. The ideal IOL would be one that mimics the natural crystalline lens at a point in life where there is excellent transmission and adequate absorption, thus balancing the need for visual performance and retinal protection. Where the spectral transmission curve should be is still the subject of ongoing debate. BFIOLs allow significantly more light into the eye than the naturally ageing crystalline lens. How well an IOL transmits and absorbs light will have an impact on its performance and its safety profile. The mechanisms of light-induced retinal toxicity are reviewed in Van Norren's article in this edition and will not be reviewed here (Van Norren-Cambridge Symposium Paper). Although there is debate about whether chronic light exposure triggers or aggravates AMD, laboratory and animal experimental studies have shown that shortwavelength light, particularly UV light, but also blue light can cause damage to the retina. 6,7 (Van NorrenCambridge Symposium Paper) There is good evidence from animal and cell culture studies that a yellow lens can protect against damage from light. ${ }^{25-31}$ Tanito et al. measured the spectral transmission of 54 IOLs including BFIOLs and UVFIOLs and estimated the retinal hazard indices. Retinal hazard indices included blue light irradiance value (characterised by an action spectrum called the hazard function, which represents the relative weight of each wavelength regarding its potential to cause damage to the retina) and maximal permissible exposure duration per day $\left(t_{\max }\right)$ for viewing sunlight. The values for $t_{\max }$ were calculated for aphakia and phakic eyes based on specific guidelines, and for pseudophakic eyes using the percentage transmittance data for the IOLs used in their study. Their study showed that compared with aphakic eyes UVFIOL's conferred a $60 \%$ reduction in the blue light irradiance value and the BFIOL reduced this further. ${ }^{32}$

\section{Influence on visual acuity}

Clinical studies comparing BFIOL and UVFIOL have found no difference in clinical performance for visual acuity. ${ }^{3} \mathrm{~A}$ meta-analysis of eight studies looking at postoperative best corrected visual acuity (BCVA) by Zhu et al ${ }^{4}$ showed no significant difference between the two groups.

\section{Influence on scotopic and photopic vision}

One of the major concerns raised regarding the use of a BFIOL rather than a UVFIOL was that visual performance in scotopic or low mesopic conditions might be impaired. $15,16,33$ As even in the healthy eye there is an agerelated decline in rods with a slowing of rod-mediated dark adaptation, ${ }^{34-38}$ anything that theoretically may 
exacerbate this should be avoided, particularly as poor dark adaptation has been associated with an increased risk for falls in an elderly population with no evident ocular pathology. 39

In addition, in eyes with age-related macular degeneration rods are preferentially lost from the parafovea, and rod function is significantly affected. ${ }^{40-42}$ Although experimental data have been published suggesting that the use of a BFIOL could reduce scotopic sensitivity by $14-25 \% .{ }^{15,43}$ Subsequent further analysis disputing this original observation was carried out, and by correcting the transmission curve and applying aphakic rather than an aphakic scoptic spectral sensitivity curve, both the UVFIOL and the BFIOL were associated with significant increase in scotopic spectral sensitivity and light available, compared with phakic young subjects. ${ }^{44}$ Futhermore clinical, non-theoretical studies investigating scotopic sensitivity did not find any difference between these two IOL groups. ${ }^{45,46}$ A study was carried out by Kiser et al, using an external filter with simulated blue-light filtering in patients with bilateral pseudophakia with UVFIOLs whose average age was 81 years old, and who had early AMD. Their performance was tested by observation of walking through two different mobility courses in scotopic conditions. There was no significant difference in performance between those wearing no filter, or wearing the wrap around blue filter. ${ }^{47}$

\section{Influence on glare, and impact on clinical assessments}

An advantage claimed for BFIOLs is better performance in conditions of glare. ${ }^{48-50}$ Gray et al evaluated the performance of 33 patients in a driving simulator, 18 of whom had BFIOLs and 15 with UVFIOLs. Those with a BFIOL were noted to have significantly lower glare susceptibility and greater safety margins than those with a UVFIOL. However, the patients with the BFIOL also had a toric correction, which the UVFIOL patients did not, which may have had an impact, although they were all fully refracted. ${ }^{48}$

A recent study reported this year by Hammond et al examined visual performance in a randomised masked cross-over clinical study in pseudophakic patients with UVFIOL's. The photostress time was assessed in 154 participants both with clear and with blue-filtering glasses after intense light exposure. Photostress recovery time and glare disability thresholds were significantly improved when participants used blue-filtering glasses compared with clear non-filtering glasses. He concludes that BFIOL's may be beneficial under intense lighting conditions. ${ }^{50}$

Several studies have investigated the impact of a blue light filter on clinical measurements including nerve fibre layer assessments, visual fields, visual-evoked potentials, and imaging of the retina during surgery. ${ }^{51-54} \mathrm{No}$ significant perioperative differences in RNFL measurements or signal strength between yellow and clear IOL were noted using the Cirrus SD-OCT. ${ }^{51} \mathrm{Kim}$ et al investigated the effect of BFIOLs on frequencydoubling technique perimetry, and found no significant difference in patients who received BFIOL from those who had clear IOLs. ${ }^{52}$ Hoffman et al's study evaluated 20 patients by obtaining multifocal VEPs for the blue light and neutral filtering conditions at all eccentricities. They found that no significant effects on amplitudes were obtained, and concluded that the use of BFIOL did not affect the visual processing. ${ }^{53}$ Falkner-Radler et al reported that BFIOLs did not represent an impediment to vitreoretinal surgery, diagnosis, or treatment compared with the clear UV-filter IOLs. They also reported that subsequent to their clinical trial they routinely use BFIOLs for all patients older than 50 years. ${ }^{54}$

\section{Influence on blood barrier disruption}

Miyake and co-authors reported that eyes with a UV or BFIOL had a lower incidence of BRB disruption than eyes with an untreated IOL (one without UV or BF filtering).

They took into account patient's age and used two clinical signs: the fluorescein concentration at the posterior vitreous and amount of autofluorescence at the posterior polar retina to evaluate the physiological state of the retina in longstanding pseudophakic eyes. They also noted that autofluorescence increased with age, even in eyes with UV-filtering IOLs and recommended that not only the UV light rays but also near-UV and blue light should be prevented from reaching the retina. ${ }^{55}$

\section{Influence on colour perception, colour vision, and colour contrast sensitivity}

It is widely known that a number of patients who receive clear lenses report that the visual scene has a blue tint (cyanopsia) post-operatively. Several studies have evaluated patient awareness of cyanopsia postoperatively comparing BFIOLs to UVFIOL. Hayashi et al ${ }^{56}$ reported that the incidence of cyanopsia was less in a BFIOL group compared with an UVFIOL group, but interestingly by 3 months neither group noticed cyanopsia, which suggests adaptation over time. In Zhu and co-authors' meta-analysis of 11 studies comparing BFIOL and UVFIOL, they noted that overall BFIOLs reduced the incidence of photophobia and cyanopsia in the early post-operative period. ${ }^{4}$ In one series after 3 months, the patients were unable to identify which eye had received which IOL implantation. ${ }^{57}$ In one case where the BFIOL was explanted owing to colour perception 
variation, the IOL was explanted just under 3 months had elapsed, and it might be that in time adaptation could have occurred. ${ }^{58}$

It has been argued that a BFIOL would increase visual quality by reducing longitudinal chromatic aberration. Siedelecki ${ }^{59}$ showed that the chromatic aberration was three times higher with clear UV-blocking IOLs compared with the human crystalline lenses. The majority of studies have showed that there is no difference between the two IOLs for colour vision or contrast sensitivity and the 11 clinical trials meta-analysed by Zhu comparing BFIOL and UVFIOL colour vision in photopic conditions including colour perception and colour contrast sensitivity post-operatively showed that both types of IOL had the same performance. The only exception was that the UVFIOL performed better under mesopic light conditions for colour vision in the blue light spectrum. ${ }^{4}$ A very recent study by Bandyopadhyay et al reported their findings in patients who received BFIOLs with different tints. In this study 33 patients received a BFIOyellow type; 33 received a BFIOL-orange type, and 33 had a UVFIOL. They reported that yellow- or orange-coloured BFIOLs were comparable to clear IOLs in terms of photopic and mesopic contrast sensitivity. ${ }^{60}$ Yuan et al in 2004 carried out a randomised controlled clinical trial in 60 patients with cataracts who received BFIOLs compared with 30 patients receiving UVFIOL's. The BFIOL group was noted to have significantly improved contrast sensitivity in the lower to middle frequencies without affecting color vision, and the incidence of photophobia and cyanopsia was less in this group. ${ }^{61}$ Niwa et al ${ }^{62}$ showed that the BFIOL is associated with better contrast sensitivity in the middle spatial frequencies of 6 and $12 \mathrm{c} / \mathrm{deg}$ in photopic and mesopic vision. In addition, the yellow-tinted IOL decreased the effect of central glare on the contrast sensitivity.

Raj et $a l^{63}$ reported that in individuals with partial red green colour vision defects that their colour vision was not worsened by implantation of a BFIOL.

\section{Age-related macular degeneration and IOL type}

The controversial subject of whether AMD is triggered or exacerbated by cataract surgery is covered in other review articles and there has been little new information to add. ${ }^{1-3}$ However, there is good evidence from animal and cell culture studies that a BFIOL can exert a protective effect. ${ }^{25-31}$ Despite the potential for damage demonstrated by the results of these studies, it has been said that these findings may not be directly applicable to the development or progression of AMD in humans. More recently, small early studies regarding the use of BFIOLs in humans relevant to this question have been published. Nagai et $a^{22}$ looked at a small group of patients who had undergone fundus autofluorescence imaging pre cataract surgery and at 2 years follow-up and noted that there was less-abnormal AF with BFIOLs (52 eyes) compared with UVFIOLs (79 eyes) and stated that the incidence of AMD was lower in the BFIOL group. Lavric and Pompe report their 2-year follow-up of 30 patients who received a BFIOL in one eye and a UVFIOL in their other eye. No statistical difference was seen between the two groups who were examined for macular changes. ${ }^{64}$ Pipis et al in 2015 report a much slower progression of geographic atrophy (GA) in the BFIOL group compared with the UVFIOL group, when they examined 66 eyes from 40 patients. Of those 27 were implanted with a BFIOL and 39 with a UVFIOL. Spectral domain optical coherence tomography and advanced RPE software analysis was used to measure lesion size and any progression over one year. There was a statistically significant difference comparing progression of GA area between the BFIOL and UVFIOL, suggesting a protective effect from the BFIOL. ${ }^{21}$

\section{Influence on circadian rhythm, in particular the sleep-wake cycle}

Mainster raised the concern that implanting a BFIOL would disrupt circadian rhythms by preventing shortwavelength light from reaching the retina. ${ }^{15}$ As light does not just stimulate the activity of photoreceptors, but also the directly photosensitive retinal ganglion cells (pRGCs), which are maximally sensitive to light at $\sim 480 \mathrm{~nm}$, and because BFIOLs filter blue light, concerns were raised that such IOLs may cause disruption of the sleep-wake cycle. The pRGCs project to multiple structures in the brain, including the suprachiasmatic nuclei (SCN) of the anterior hypothalamus, which act as the master circadian pacemaker. The SCN in turn regulate circadian biology throughout the body. ${ }^{17,65}$ Ocular light input is required to synchronise the body clock to the environmental daynight cycle. ${ }^{17}$

It has already been shown in a number of studies that in addition to improving vision, cataract surgery has been shown to lead to an improved sleep-wake pattern. ${ }^{17-19,66}$

This benefit is presumed due to the increased amount of light reaching the retina. Landers et al ${ }^{66}$ carried out a cross-sectional study comparing the sleep quality of 31 individuals with UVB-IOL and 18 individuals with BFIOL. They showed that PSQI-derived sleep quality did not differ between UVB-IOL and BFIOL lens types.

Espindle et al, although they did not assess sleep, investigated health-related quality of life in 257 patients, who received either a UV-IOL or a BFIOL after cataract surgery using a vision-specific (NEI VFQ-39) and a generic physical and mental health (SF-12) scale. They reported an improvement in vision-related functions in 
both groups after surgery, but no difference between those with bilateral UVB-IOL implants and those with bilateral BFIOL implants. ${ }^{67}$ Alexander et al in a large prospective, consecutive, dual-site study, with 961 participants completing the study, showed no difference between the BFIOL and UV-IOL groups. Sleep quality improved significantly following cataract surgery in the short term for the entire cohort, irrespective of the type of lens implanted. ${ }^{17}$ Schmoll et al ${ }^{18}$ looked at sleep-wake and cognitive reaction times and showed no difference between BFIOL or UVFIOL. The CHUKYO study was designed to evaluate blood pressure and sleep duration after IOL implantation during cataract surgery and to compare different types of IOL. Using a questionnaire, as well as obtaining blood pressure measurements and sleep duration, they obtained data from 1367 patients (1367 eyes) before IOL implantation, 1 week after IOL implantation and 1 month after IOL implantation. They reported that the diastolic blood pressure was significantly decreased in the total patient group after implantation. The decrease in systolic blood pressure 1 month after implantation was significantly more in patients who received a BFIOL than it was in those who received an UVFIOL. They suggested that a BFIOL is better for patients with high blood pressure than a UVFIOL. In addition, they reported that there was no difference between the BFIOL, or the UVFIOL in improving sleep duration. They reported no significant differences in vision among the IOL groups, but they suggested that differences in spectral transmittance properties might have resulted in different effects on the autonomic nervous system and melatonin secretion. They explain the greater decrease in blood pressure in patients with BFIOLs may be because blood pressure is known to increase with blue light exposure in people with normal blood pressure. ${ }^{68}$ Thus, blue light, which was not filtered by UV light-filtering IOLs, may counteract the blood pressure reduction after IOL implantation. ${ }^{19}$ Leruez carried out a pilot study in patients who had had BFIOL or UV-IOL implants to determine whether implantation with BFIOLs would influence post-operative mood, or induce more depression compared with UVFIOL. They found no difference between BFIOL and UVFIOL. Mood improved in both groups after surgery. ${ }^{20}$

\section{Discussion}

There are a number of advances in IOL technology, which can make the choice of an IOL complex and challenging. In the ongoing debate whether to implant a BFIOL or a clear IOL, there is now clear evidence to show that the visual performance with both types of IOL is similar. Clinical studies have shown that visual acuity, photopic and scotopic, and colour vision performance is essentially the same with BFIOL and UVFIOL implants. ${ }^{1-5,9,44,45,69}$

The concerns that clinical imaging and assessment and visual field testing may be affected by implantation of a BFIOL have been shown not to be the case.

It was also suggested that circadian rhythm, in particular sleep, could be disrupted using a BFIOL but this concern has also been dispelled. ${ }^{17-19,66}$ Cataract surgery for the main part is carried out in an elderly population in whom there is an age-related decline in retinal photoreceptors and ganglion cell populations, as well as the function of the circadian clock, and with ageing smaller pupils. It is clear that access to light is important for this group. Pupil size and access to light have a more important role than the current IOLs. ${ }^{17}$

Overall, it would seem that there are no drawbacks to using a BFIOL, and indeed there are benefits of using an IOL which filters out short-wavelength light including reduction in glare. ${ }^{11,48-50}$ However, more importantly as short-wavelength light has been shown to be toxic to the retina and results of in vitro, animal and clinical studies support the hypothesis that protecting the retina from short-wavelength light is desirable, implantation with a BFIOL seems a sensible precaution. (Van NorrenCambridge Symposium Paper) ${ }^{6,7,25-31}$ Early evidence in a small study has shown that BFIOLs have a potential to reduce the advance of $\mathrm{GA}^{21}$ and there is also emerging evidence that short-wavelength light may have a role in ocular melanoma development, ${ }^{70}$ as well as skin melanomas, making protection from short-wavelength light desirable for the eye. The paediatric population that have a longer exposure to short-wavelength light may be best served by a BFIOL implantation. ${ }^{3}$ It is important to advise individuals who have had a UVFIOL implant that they should wear BF sunglasses in sunlight. It is also probably best to avoid implanting a BFIOL into one eye and a UVFIOL into the other, as Schweitzer showed that six patients noticed a disparity in colour perception between each eye. ${ }^{71}$ There are a wide range of IOLs available and it is not within the scope of this article to specifically discuss any particular IOL. However, it is important that the IOL design allows photoreception as close as possible to the young adult healthy crystalline lens, and photoprotection to prevent short-wavelength light damage over the lifetime of the recipient.

In summary, there is good evidence in the literature that implantation of a BFIOL does not impair visual acuity, photopic, scotopic, or colour vision, nor does it affect the sleep-wake cycle. In addition, there are some definite and theoretical benefits associated with implanting a BFIOL, including improved performance in glare but more importantly protection of the retina against short-wavelength light. 


\section{Conflict of interest}

The author declares no conflict of interest.

\section{Acknowledgements}

This work was part funded from a Wellcome Trust Grant (047473). The author is funded by the Oxford Biomedical Centre of Excellence.

\section{References}

1 Cuthbertson FM, Peirson SN, Wulff K, Foster RG, Downes $\mathrm{SM}$. Blue light-filtering intraocular lenses: review of potential benefits and side effects. J Cataract Refract Surg 2009; 35(7): 1281-1297.

2 Henderson BA, Grimes KJ. Blue-blocking IOL's: a complete review of the literature. Surv Ophthalmol 55: 284-289; 2010.

3 Davison JA, Patel AS, Cunha JP, Schwiegerling J, Muftuoglu $\mathrm{O}$. Recent studies provide an updated clinical perspective on blue light-filtering IOLs. Graefes Arch Clin Exp Ophthalmol 2011; 249: 957-968.

4 Zhu XF, Zou HD, Yu YF, Sun Q, Zhao NQ. Comparison of blue light-filtering IOLs and UV light-filtering IOLs for cataract surgery: a meta-analysis. PLoS One 2012; 7(3): e33013.

5 Ardjomand N, Wirtisch MG, Wenzl E. Yellow intraocular lenses - to block or not to block. Eur Ophthal Rev 2009; 3(2): 77-79.

6 Ham Jr WT, Mueller HA, Sliney DH. Retinal sensitivity to damage from short wavelength light. Nature 1976; 260: 153-155.

7 Noell WK, Walker VS, Kang BS, Berman S. Retinal damage by light in rats. Invest Ophthalmol 1966; 5: 450-473.

8 Margrain TH, Boulton M, Marshall J, Sliney DH. Do blue light filters confer protection against age-related macular degeneration? Prog Retin Eye Res 2004; 23: 523-553.

9 Wolffsohn JS, Cochrane AL, Khoo H, Yoshimitsu Y, Wu S. Contrast is enhanced by yellow lenses because of selective reduction of short-wavelength light. Optom Vis Sci 2000; 77: 73-81.

10 Hammond Jr BR, Renzi LM, Sachak S, Brint SF. Contralateral comparison of blue-filtering and non-bluefiltering intraocular lenses: glare disability, heterochromatic contrast, and photostress recovery. Clin Ophthalmol 2010; 4 (1): 1465-1473.

11 Simunovic MP. On seeing yellow: the case for, and against, short-wavelength light-absorbing intraocular lenses. Arch Ophthalmol 2012; 130(7): 919-926.

12 Miyake K, Ichihashi S, Shibuya Y, Ota I, Miyake S, Terasaki $\mathrm{H}$. Blood-retinal barrier and autofluorescence of the posterior polar retina in long-standing pseudophakia. J Cataract Refract Surg 1999; 25(7): 891-897.

13 Kondrashev SL. Long-wave sensitivity in the masked greenling (Hexagrammos octogrammus), a shallow-water marine fish. Vis Res 2008; 48: 2269-227.

14 Wenzl E, Wohlfart C, Vidic B, Zalaudek K, El-Shabrawi Y, Wirtitsch MG et al. Colour vision and contrast sensitivity with photochromatic IOL. Spektrum derAugenheilkunde 2009; 23: 17-20.

15 Mainster MA. Violet and blue light blocking intraocular lenses: photoprotection versus photoreception. $\mathrm{Br} J$ Ophthalmol 2006; 90: 784-792.
16 Mainster MA, Turner PL. Blue-blocking IOLs decrease photoreception without providing significant photoprotection (viewpoints). Surv Ophthalmol 2010; 55: 272-289.

17 Alexander I, Cuthbertson FM, Ratnarajan G, Safa R, Mellington FE, Foster RG et al. Impact of cataract surgery on sleep in patients receiving either ultraviolet-blocking or bluefiltering intraocular lens implants. Invest Ophthalmol Vis Sci 2014; 55(8): 4999-5004.

18 Schmoll C, Khan A, Aspinall P, Goudie C, Koay P, Tendo $C$ et al. New light for old eyes: comparing melanopsin-mediated non-visual benefits of blue-light and UV blocking intraocular lenses. Br J Ophthalmol 2014; 98(1): 124-128.

19 Ichikawa KCHUKYO study investigators. Changes in blood pressure and sleep duration in patients with blue lightblocking/yellow-tinted intraocular lens (CHUKYO study). Hypertens Res 2014; 37(7): 659-664.

20 Leruez S, Annwiler C, Gohier B, Beauchet O, Ebran MJ, Gohier $\mathrm{P}$ et al. Blue light-filtering intraocular lenses and postoperative mood: a pilot clinical study. Int Ophthalmol 2015; 35(2): 249-256.

21 Pipis A, Touliou E, Pillunat LE, Augustin AJ. Effect of the blue filter intraocular lens on the progression of geographic atrophy. Eur J Ophthalmol 2015; 25(2): 128-133. 2014.

22 Nagai H, Hirano Y, Yasukawa T, Morita H, Nozaki M, Wolf-Schnurrbusch U et al. Prevention of increased abnormal fundus autofluorescence with blue light-filtering intraocular lenses. J Cataract Refract Surg 2015; 41(9): 1855-1859.

23 Artigas JM, Felipe A, Navea A, Artigas C, Garcia Domene MC. Spectral transmittance of intraocular lenses under natural and artificial illumination -criteria analysis for choosing a suitable filter. Ophthalmology 2011; 118: 3-8.

24 van Norren D, van der Kraats J. Spectral transmission of intraocular lenses expressed as a virtual age. $\mathrm{Br} \mathrm{J}$ Ophthalmol 2007; 91: 1374-1375.

25 Nilsson SEG, Textorius O, Andersson B-E, Swenson B. ClearPMMA versus yellow intraocular lens material. An electrophysiologic study on pigmented rabbits regarding 'the blue light hazard'. Prog Clin Biol Res 1989; 314: 539-553.

26 Sparrow JR, Miller AS, Zhou J. Blue light-absorbing intraocular lens and retinal pigment epithelium protection in vitro. J Cataract Refract Surg 2004; 30: 873-878.

27 Rezai KA, Gasyna E, Seagle B-LL, Norris Jr JR, Rezaei KA. AcrySof Natural filter decreases blue light-induced apoptosis in human retinal pigment epithelium. Graefes Arch Clin Exp Ophthalmol 2008; 246: 671-676.

28 Yanagi $Y$, Inoue $Y$, Iriyama A, Jang W-D. Effects of yellow intraocular lenses on light-induced upregulation of vascular endothelial growth factor. J Cataract Refract Surg 2006; 32: 1540-154.

29 Kent M, Neubauer AS, Liegl R, Eibl KH, Alge CS, Lackerbauer CA et al. Cytoprotective effects of a blue lightfiltering intraocular lens on human retinal pigment epithelium by reducing phototoxic effects on vascular endothelial growth factor alpha, Bax, and Bel-2 expression. J Cataract Refract Surg 2009; 35: 354-362.

30 Augustin AJ. Reliable UV-light protection in intraocular lenses-scientific rationale and quality requirements. Klin Monbl Augenheilkd 2014; 231(9): 901-908.

31 Tanito M, Kaidzu S, Anderson RE. Protective effects of soft acrylic yellow filter against blue light-induced retinal damage in rats. Exp Eye Res 2006; 83(6): 1493-1504.

32 Tanito M, Okuno T, Ishiba Y, Ohihara A. Transmission spectrum and retinal blue-light irradiance values of untinted 
and yellow-tinted intraocular clenses. I Cataract Refract Surg 2010; 36(2): 299-307.

33 Mainster MA. Intraocular lenses should block UV radiation and violet but not blue light. Arch Ophthalmol 2005; 123: 550-555.

34 Jackson GR, Owsley C, McGwin Jr G. Aging and dark adaptation. Vis Res 1999; 39: 3975-3982.

35 Jackson GR, Owsley C, Cordle EP, Finley CD. Aging and scotopic sensitivity. Vis Res 1998; 38: 3655-3662.

36 Jackson GR, Owsley C. Scotopic sensitivity during adulthood. Vis Res 2000; 40: 2467-2473.

37 Schefrin BE, Tregear SJ, Harvey Jr LO, Werner JS. Senescent changes in scotopic contrast sensitivity. Vis Res 1999; 39: 3728-3736.

38 Gunkel RD, Gouras P. Changes in scotopic visibility thresholds with age. Arch Ophthalmol 1963; 69: 4-9.

39 McMurdo MET, Gaskell A. Dark adaptation and falls in the elderly. Gerontology 1991; 37: 221-222.

40 Jackson GR, Owsley C, Curcio CA. Photoreceptor degeneration and dysfunction in aging and age-related maculopathy. Ageing Res Rev 2002; 1: 381-39.

41 Owsley C, Jackson GR, Cideciyan AV, Huang Y, Fine SL, Ho AC et al. Psychophysical evidence for rod vulnerability in age-related macular degeneration. Invest Ophthalmol Vis Sci 2000; 41: 267-273.

42 Sunness JS, Rubin GS, Applegate CA, Bressler NM, Marsh MJ, Hawkins BS et al. Visual function abnormalities and prognosis in eyes with age-related geographic atrophy of the macula and good visual acuity. Ophthalmology 1997; 104: 1677-1691.

43 Mainster M, Sparrow JR. How much blue light should an IOL transmit? Br J Ophthalmol 2003; 87: 1523-1529.

44 Schwiegerling J2009Blue light-filtering intraocular lenses and scotopic sensitivity. J Cataract Refract Surg 35: 2032.

45 Greenstein VC, Chiosi F, Baker P, Seiple W, Holopigian K, Braunstein RE et al. Scotopic sensitivity and color vision with a blue-light-absorbing intraocular lens. J Cataract Refract Surg 2007; 33 (4): 667-672.

46 Muftuoglu O, Karel F, Duman R. Effect of a yellow intraocular lens on scotopic vision, glare disability, and blue color perception. J Cataract Refract Surg 2007; 33(4): 658-666.

47 Kiser AK, Deschler EK, Dagnelie G. Visual function and performance with blue-light blocking filters in age related macular degeneration. Clin Experiment Ophthalmol 2008; 36: 514-520.

48 Gray R, Hill W, Neuman B, Houtman D, Potvin R. Effects of a blue light-filtering intraocular lens on driving safety in glare conditions. J Cataract Refract Surg 2012; 38(5): 816-822.

49 Gray R, Perkins SA, Suryakumar R, Neuman B, Maxwell WA. Reduced effect of glare disability on driving performance in patients with blue light-filtering intraocular lenses. J Cataract Refract Surg 2011; 37(1): 38-44.

50 Hammond BR, Antonio B. Attenuating photostress and glare disability in pseudophakic patients through the addition of a short-wave absorbing filter. J Ophthalmol 2015; 2015: 607635.

51 Kim JH, Kim NR, Lee ES, Rho S, Kang SY, Kim CY. Influence of blue light-filtering intraocular lenses on retinal nerve fiber layer measurements by spectral-domain optical coherence tomography. Curr Eye Res 2011; 36(10): 937-942.

52 Kim JY, Choi JA, Na KS, Joo CK. The effect of yellow tinted intraocular lenses on the result of frequency doubling perimetry after cataract surgery. Korean J Ophthalmol 2011; 25(1): 29-32.

53 Hoffmann MB, Behrens-Baumann W, Langenbucher A, Reupsch J, Viestenz A. Effect of blue-light filtering on multifocal visual-evoked potentials. I Cataract Refract Surg 2012; 38(1): 85-91.

54 Falkner-Radler CI, Benesch T, Binder S. Bluelight-filter intraocular lenses in vitrectomy combined with cataract surgery: results of a randomized controlled clinical trial. Am J Ophthalmol 2008; 145: 499-503.

55 Miyake K, Ichihashi S, Shibuya Y, Ota I, Miyake S, Terasaki $\mathrm{H}$. Blood-retinal barrier and autofluorescence of the posterior polar retina in long-standing pseudophakia. J Cataract Refract Surg 1999; 25(7): 891-897.

56 Hayashi K, Hayashi H. Visual function in patients with yellow tinted intraocular lenses compared with vision in patients with non-tinted intraocular lenses. $\mathrm{Br} J$ Ophthalmol 2006; 90(8): 1019-1023.

57 Olson MD, Miller KM. Implanting a clear intraocular lens in one eye and a yellow lens in the other eye: a case series. Am J Ophthalmol 2006; 141(5): 957-958.

58 Shah SA, Miller KM. Explantation of an AcrySof Natural intraocular lens because of a color vision disturbance. Am J Ophthalmol 2005; 140(5): 941-942.

59 Siedlecki D, Ginis HS. On the longitudinal chromatic aberration of the intraocular lenses. Optom Vis Sci 2007; 84: 984-989.

60 Bandyopadhyay S, Saha M, Chakrabarti A, Sinha A. Effect on contrast sensitivity after clear, yellow and orange intraocular lens implantation. Int Ophthalmol 2015; (Epub ahead of print).

61 Yuan Z, Reinach P, Yuan J. Contrast sensitivity and color vision with a yellow intraocular lens. Am J Ophthalmol 2004; 138(1): 138-140.

62 Niwa K, Yoshino Y, Okuyama F, Tokoro T. Effects of tinted intraocular lens on contrast sensitivity. Ophthalmic Physiol Opt 1996; 16: 297-302.

63 Raj SM, Vasavada AR, Nanavaty MA. AcrySof Natural SN60AT versus AcrySof SA60AT intraocular lens in patients with color vision defects. J Cataract Refract Surg 2005; 31(12): 2324-2328.

64 Lavric A, Pompe MT. Do blue-light filtering intraocular lenses affect visual function? Optom Vis Sci 2014; 91(11): 1348-1354.

65 Foster RG, Hankins MW. Circadian vision. Curr Biol 2007; 17: R746-R751.

66 Landers JL, Tamblyn D, Perriam D. Effect of a blue-lightblocking intraocular lens on the quality of sleep. J Cataract Refract Surg 2009; 35: 83-88.

67 Espindle D, Crawford B, Maxwell A, Rajagopalan K, Barnes R, Harris B et al. Quality-of-life improvements in cataract patients with bilateral blue light-filtering intraocular lenses: clinical trial. J Cataract Refract surg 2005; 31(10): 1952-1959.

68 Yasukouchi A, Ishibashi K. Non-visual effects of the color temperature of fluorescent lamps on physiological aspects in humans. J Physiol Anthropol Appl Human Sci 2005; 24: $41-43$.

69 Yuan Z, Reinach P, Yuan J. Contrast sensitivity and color vision with a yellow intraocular len. Am J Ophthalmol 2004; 138(1): 138-140.

70 Mallet JD, Gendron SP, Drigeard DMC, Rochette PJ. Implication of ultraviolet light in the etiology of uveal melanoma a review. Photochem Photobiol 2013; doi:10.1111/ php.12161.

71 Schweitzer C, Colin J. Patient-reported difference following implantation of a blue light-filtering aspheric intraocular lens and a uv-filtering aspheric intraocular lens. Case Rep Ophthalmol 2013; 4(3): 248-251. 